\title{
Spectroscopic studies on the interaction of bilirubin with symmetrical alkyl diamines
}

\author{
KASTURI LAHIRI PURANAM and P. BALARAM* \\ Molecular Biophysics Unit, Indian Institute of Science, Bangalore 560 012, India
}

\begin{abstract}
The interactions of symmetrical alkyldiamines with bilirubin-IX $\alpha$ have been examined in dichloromethane and dioxane solutions, by visible region difference spectroscopy and florescence methods. In dioxane solutions a clear difference is observed between the complexes of the shorter chain diamines (number of spacer methylene groups $(n \leq 4)$ ) and the longer chain diamines $(n \geq 6)$. The variations in spectral features with diamine chain length are less pronounced in dichloromethane. The spectroscopic results are consistent with the occurrence of distinct bilirubin conformations depending upon the solvent and the geometry of the interacting receptor. Based on molecular modelling two conformations are proposed. A 'ridge-tile' model similar to that observed in crystals is favoured for binding to the longer diamines, while a 'quasi-cyclic' structure is preferred for interaction with the short chain diamines.
\end{abstract}

Keywords. Bilirubin conformations; bilirubin-amine interactions; difference spectroscopy; fluorescence.

\section{Introduction}

The structure and interactions of bilirubin, a linear tetrapyrrole pigment (figure 1, represented as the predominant isomer bilirubin IX $\alpha$ ) formed by the catabolism of haemoglobin, have attracted considerable attention over the past several years (Lightner, 1982; Brodersen, 1980,1982). Disorders of bilirubin metabolism lead to a variety of clinical syndromes (Berthelot et al., 1982). The physiological importance of bilirubin interactions with plasma albumin, in transport of the pigment, has led to detailed investigations of the interactions of the pigment with albumins from humans and other animal sources (Brodersen, 1982; Blauer et al., 1977). While bilirubin-IX $\alpha$ is an optically inactive molecule, binding to chiral sites on proteins can result in induced circular dichroism, a property that has proved useful for probing pigmentprotein interactions (Blauer, 1983; Blauer and Wagniere, 1975). There have however been few attempts to delineate the conformation of bilirubin at the albumin binding

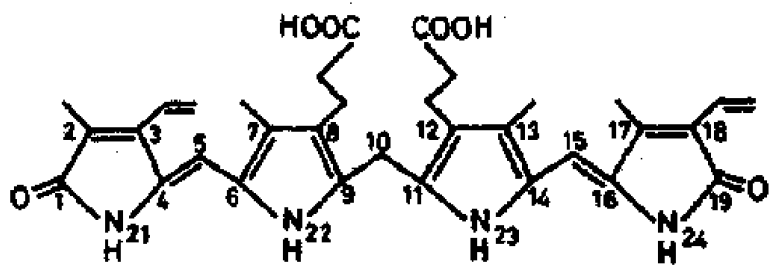

Figure 1. Structure, of bilirubin-IX $\alpha$ (4Z, $15 \mathrm{Z}$ configuration) with numbering scheme (Lightner, 1982).

*To whom all correspondence should be addressed. 
site. Indeed, even the nature of bilirubin conformations in solution have been the subject of some controversy (see, Lightner, 1982 for a succinct overview). A partially extended 'ridge-tile' structure, which has the two dipyrromethene units in an approximately orthogonal orientation has been determined by x-ray diffraction in the crystalline state (Bonnett et al., 1978). Dramatic changes in the sign of the circular dichroism bands of bilirubin bound to human serum albumin on changing $\mathrm{pH}$ or ionic strength (Brodersen, 1982; Blauer, 1983) and the differences in the circular dichroism spectra of the pigment bound to human and bovine albumins suggest that precise orientation of the interacting groups at the receptor site can determine the conformation of the bound pigment (Brodersen, 1982; Blauer, 1983). The presence of two propionic acid sidechains in bilirubin, suggests that electrostatic interactions with positively charged, basic residues may contribute to stabilizing pigment-protein complexes (Marr-Leisy et al., 1985). In this report we describe fluorescence and visible difference spectroscopic studies of the interaction of bilirubin-IX $\alpha$ with symmetrical diamine receptors in organic solvents. The results provide evidence for conformational transitions in bilirubin as a function of the solvent and the nature of the interacting 'receptor' molecule.

\section{Materials and methods}

Bilirubin-IX $\alpha$ (Sigma) was purified by a procedure adopted from that of McDonagh and Assisi (1972). Concentrations of bilirubin solutions in organic solvents were estimated using a molar extinction coefficient of $\varepsilon_{455}=62,000$ (London et al., 1983). 1,2-Diaminoethane (British Drug House), 1,6-diaminohexane (Koch-Light), 1,4diaminobutane, 1,5-diaminopentane and 1,8-diaminooctane (Sigma) were commercial samples used directly. Dioxane was purified by treatment with concentrated $\mathrm{HCl}$, drying and fractionation (Furnish et al., 1978). Dichloromethane was distilled and passed over basic alumina, prior to use. Difference spectra in visible regioin were recorded on a Shimadzu 210-A spectrophotometer fitted with a scale expander, using $1 \mathrm{~cm}$ pathlength, matched cuvettes. Fresh bilirubin solutions were used in both cuvettes to obtain a baseline in every experiment. Fluorescence spectra were recorded on a Perkin-Elmer MPF-44A spectrofluorimeter.

\section{Results and discussion}

Figure 2 shows the absorption spectrum of bilirubin-IX $\alpha$ in dioxane. An intense band at $\sim 450 \mathrm{~nm}$ is observed (Lightner, 1982). Addition of 1,2-diaminoethane or 1,2diaminohexane results in only very small changes in band shape. These effects are clearly illustrated by difference spectroscopy and the results are shown in figure 3 for the case of 1,2-diaminoethane in dioxane. Addition of increasing amounts of the diamine results in an enhancement in intensity at $\sim 480 \mathrm{~nm}$ and a larger decrease in intensity at $\sim 450 \mathrm{~nm}$. At amine: bilirubin ratios $>>2: 1$ the features of the difference spectrum change and two positive bands at $\sim 430 \mathrm{~nm}$ and $\sim 490 \mathrm{~nm}$ are observed. A plot of changes in optical density versus diamine concentration shows a large discontinuity at an amine: bilirubin ratio of 1 . Figure 4 compares the difference spectra for amines of varying chainlength, with the number of spacer methylene groups (n) ranging from $\mathrm{n}=2$ to $\mathrm{n}=8$, obtained in $\mathrm{CH}_{2} \mathrm{Cl}_{2}$ solutions at a $1: 1$ stoichiometry of 


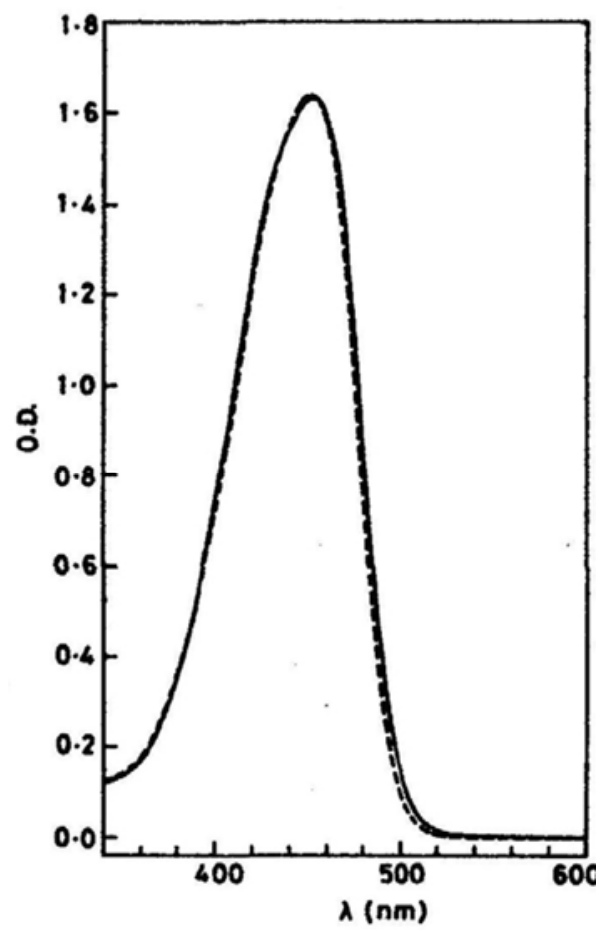

Figure 2. Absorption spectrum of bilirubin $\left(265 \times 10^{-5} \mathrm{M}\right)$ in dioxane (----). Absorption spectrum of bilirubin $\left(265 \times 10^{-5} \mathrm{M}\right)-1.2$ diaminoethane $\left(3 \cdot 0 \times 10^{-4} \mathrm{M}\right)$ in dioxane $(-)$.

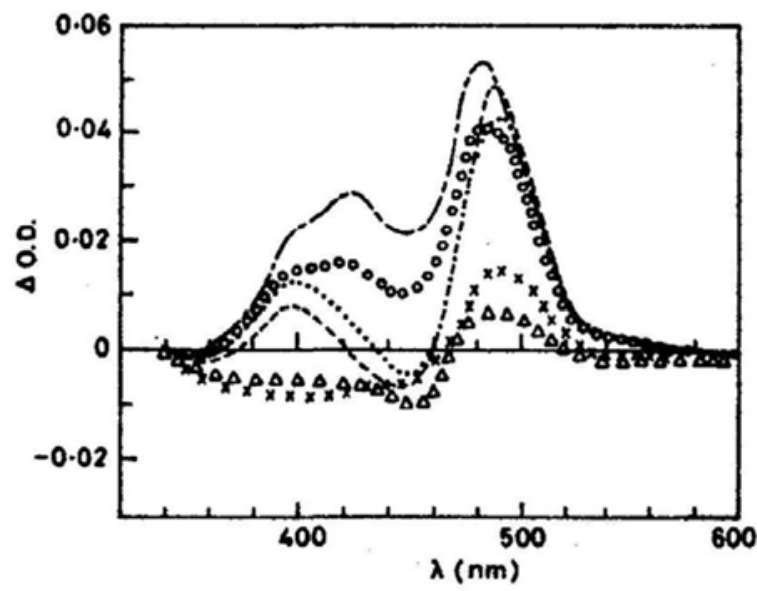

Figure 3. Visible difference spectra of bilirubin $\left(2.65 \times 10^{-5} \mathrm{M}\right)$ in the presence of increasing amounts of 1,2 diaminoethane in dioxane. The diamine to bilirubin ratios are, $(\Delta), 0 \cdot 2 ;(\times)$, $0 \cdot 45 ;(---), 1 \cdot 1 ;(.),. 1 \cdot 4 ;(\mathrm{O}), 1 \cdot 8 ;(---), 2 \cdot 0$.

the amine and bilirubin. The results of a similar study carried out in dioxane are summarized in figure 5. The difference spectra generated for the diamines with $\mathrm{n}=6$ and 8 in this solvent are dramatically different and show a large intensification at $\sim 490 \mathrm{~nm}$ and a significant loss of intensity at $450 \mathrm{~nm}$. The changes in intensity at 


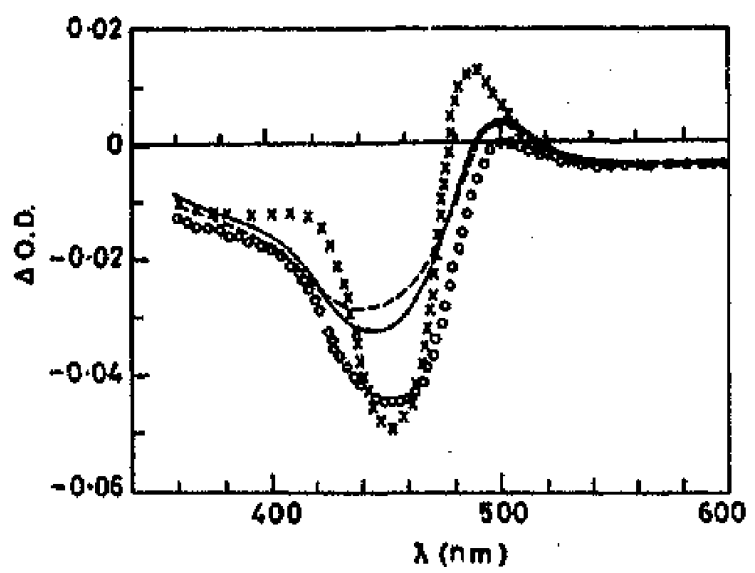

Figure 4. Visible differences spectra in dichloromethane of $1: 1$ bilirubin-diamine mixtures for the various diamines. Concentration of bilirubin is $2 \cdot 19 \times 10^{-5} \mathrm{M}$. (- - ), 1,2 diaminoethane; $(-), 1,4$ diaminobutane; $(\mathrm{O}), 1,6$ diaminohexane; $(\times), 1,8$ diaminooctane.

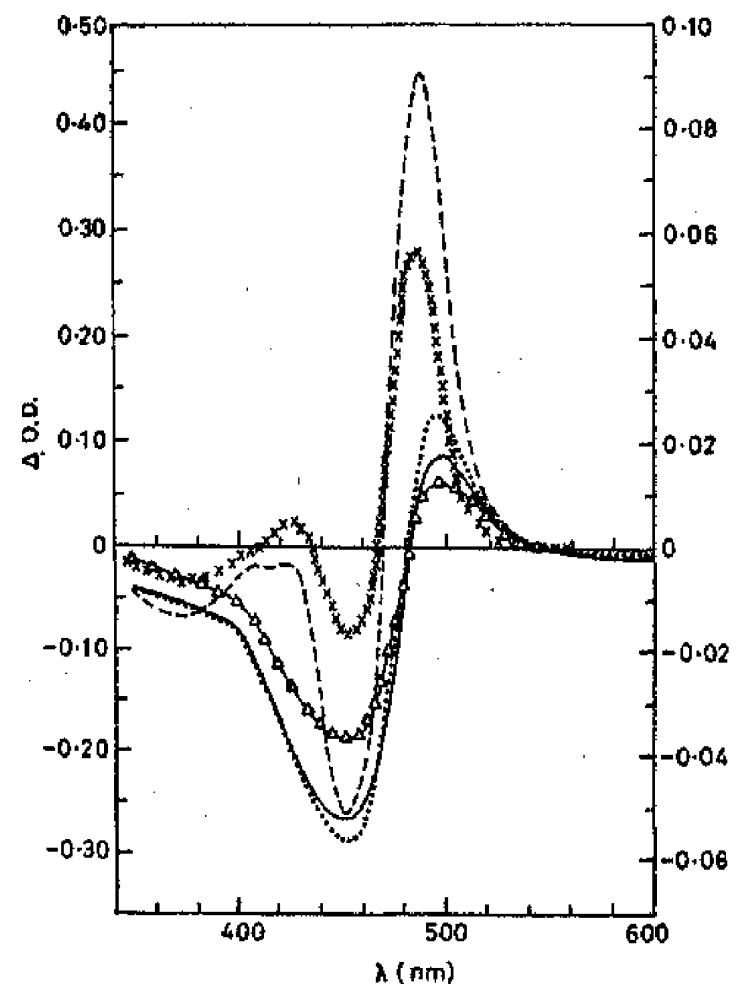

Figure 5. Visible difference specta in dioxane of $1: 1$ bilirubin-diamine mixtures for diamines of various chain lengths. Bilirubin concentration is $2.78 \times 10^{-5} \mathrm{M},(\Delta), 1,2$ diaminoethane; $(-), 1,4$ diaminobutane; (...), 1,5 diaminopentane; $(---), 1,6$ diaminohexane; $(\times), 1,8$ diaminooctane. Left hand scale corresponds to the $n=8$ amine. Note difference in intensities. 
the peak and trough values in the difference spectra are plotted against amine chain length, for the two solvents in figure 6 . In the case of dioxane a sharp discontinuity is seen between $\mathrm{n}=5$ and $\mathrm{n}=6$, while this change is less pronounced in $\mathrm{CH}_{2} \mathrm{Cl}_{2}$. The results suggest that a significant structural change in the bilirubin chromophore is induced by the altered geometry of the interacting amino groups.

Further confirmation of these observations is obtained from fluorescence spectroscopic studies. Figure 7 shows the effect of addition of 1,2-diaminohexane on the
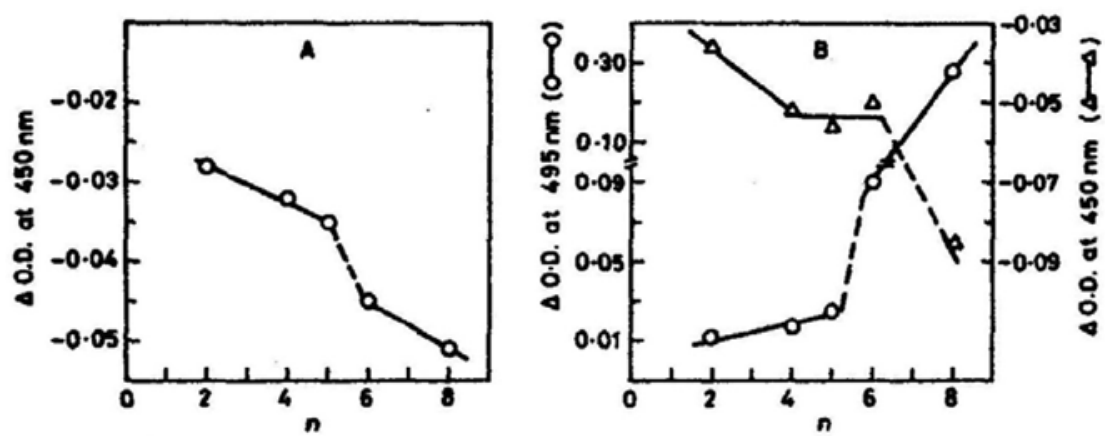

Figure 6. Dependence of the peak and trough intensities of the bilirubin-diamine difference spectra on the methylene chain length (n) of the diamines. (A), Dichloromethane; (B), dioxane.

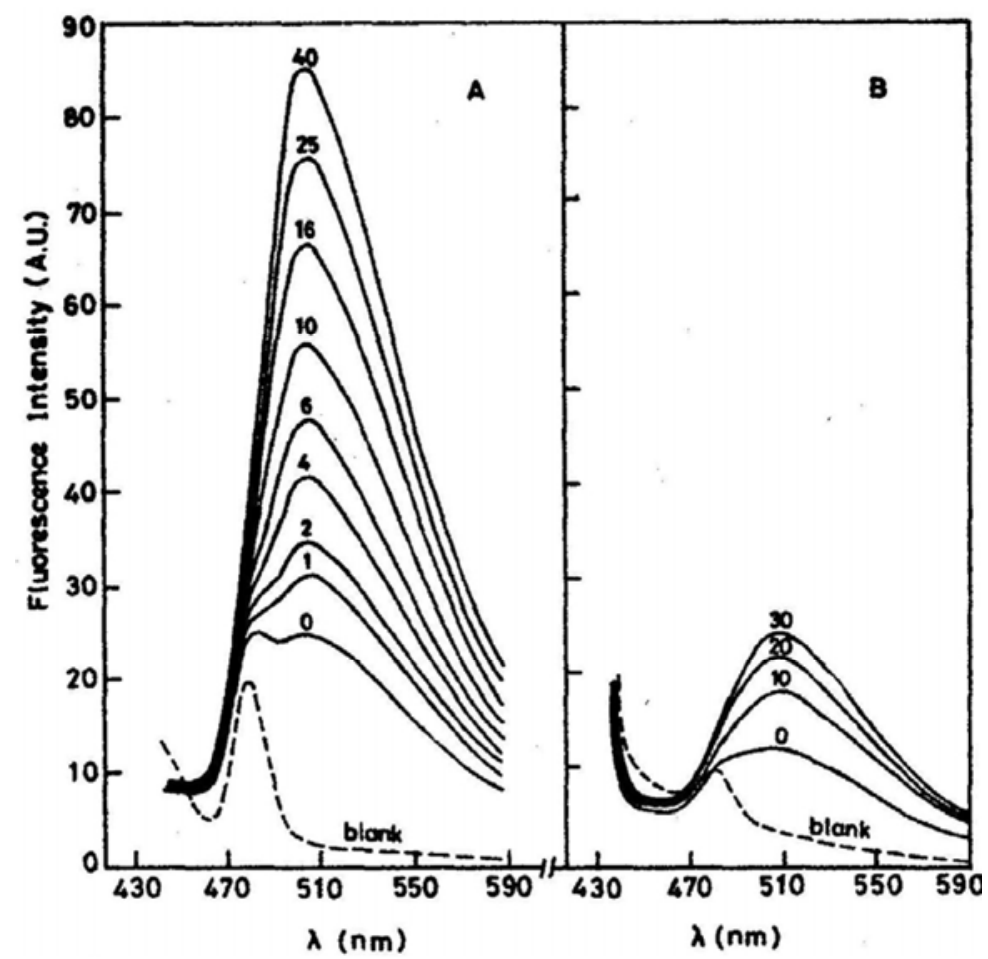

Figure 7. Enhancement of bilirubin $\left(6.53 \times 10^{-6} \mathrm{M}\right)$ fluorescence upon the addition of 1,6 diaminohexane in dioxane (A) and dichloromethane (B). The number on each trace indicates the diamine to bilirubin ratio. $\lambda_{\mathrm{ex}}=420 \mathrm{~nm}$. 
fluorescence of bilirubin in dioxane and $\mathrm{CH}_{2} \mathrm{Cl}_{2}$. Free bilirubin is weakly fluorescent in both solvents with an emission maximum at $\sim 515 \mathrm{~nm}$ on exciting at $420 \mathrm{~nm}$ (Lightner, 1982; Holzwarth et al., 1980). In dioxane, addition of 1,2-diaminohexane leads to a large enhancement in fluorescence, whereas the intensity increases are much less in dichloromethane solutions. Figure 8 compares the concentration dependence of the fluorescence enhancements observed for various diamines in dioxane. While very small effects are noted for the $n=2$ to 4 amines, the increases in intensity are larger for $\mathrm{n}=5$ and 6 , with the most significant fluorescence enhancement being observed for $\mathrm{n}=8$. In contrast, in dichloromethane solutions an enhancement of only 2.5 fold in bilirubin emission is observed even in the presence of a 30 fold excess of 1,6-diaminohexane.

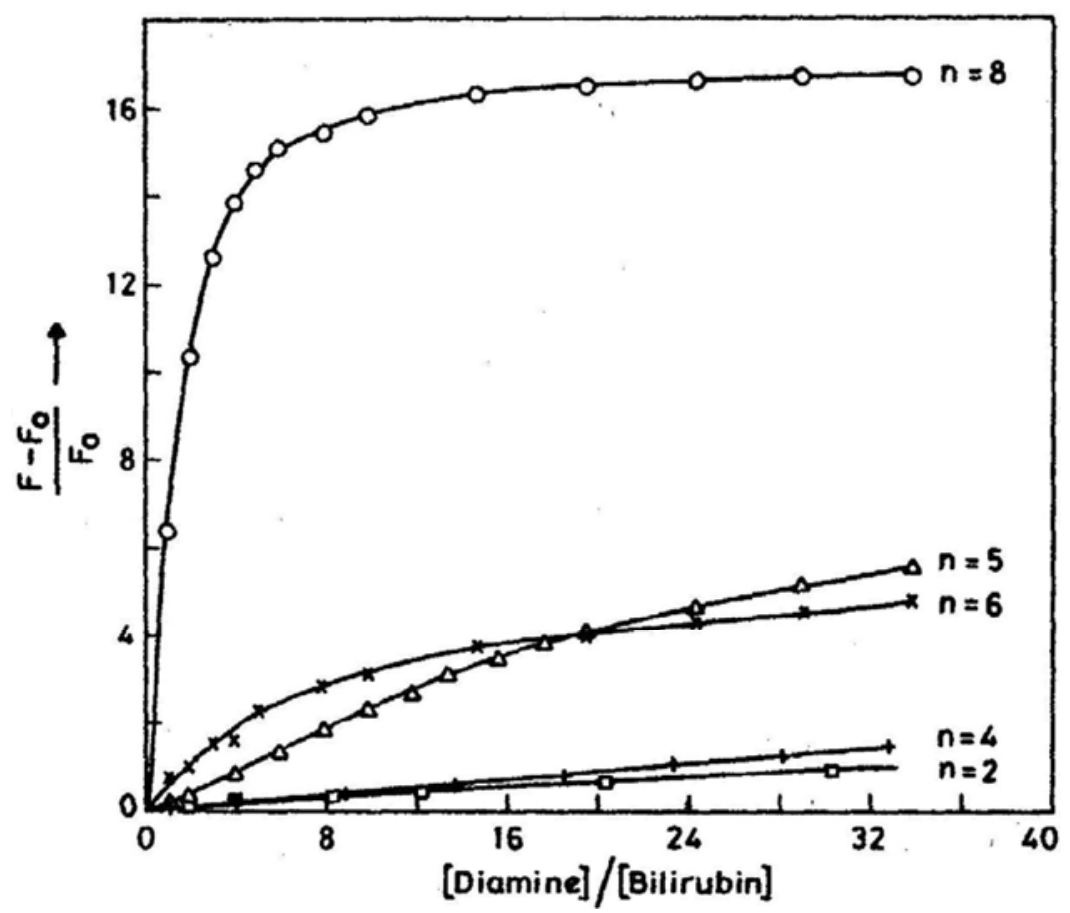

Figure 8. Enhancement of bilirubin $\left(6.53 \times 10^{-6} \mathrm{M}\right)$ fluorescence in dioxane as a function of diamine to bilirubin ratio for various diamines. $F_{o}$ is the initial bilirubin fluorescence and $F$ is the enhanced fluorescence. $\lambda_{\mathrm{ex}}=420 \mathrm{~nm}, \lambda_{\mathrm{em}}=520 \mathrm{~nm}$.

The results presented above suggest that in dioxane solutions the conformations of bilirubin in the pigment-amine complex are dependent on the methylene chain length separating the two interacting amino groups. Clearly the nature of the bilirubin species observed for the complexes with $n=2$ and $n=8$ are distinctly difference. Bilirubin-IX $\alpha$ used in the present study has the 4Z, 15Z configuration about the $\mathrm{C}_{4}-\mathrm{C}_{5}$ and $\mathrm{C}_{15}-\mathrm{C}_{16}$ double bonds (Lightner, 1982). In considering conformational flexibility, torsional freedom about the $\mathrm{C}_{5}-\mathrm{C}_{6}, \mathrm{C}_{14}-\mathrm{C}_{15}, \mathrm{C}_{9}-\mathrm{C}_{10}$ and $\mathrm{C}_{10}-\mathrm{C}_{11}$ bonds needs to be taken into account. The conformational freedom about the $\mathrm{C}_{5}-\mathrm{C}_{6}$ and $\mathrm{C}_{14}-\mathrm{C}_{15}$ bonds is limited since extensive out-of-plane distortions can disrupt $\pi$ - 
electron delocalization in the pyrrodimethene units (Geddes et al., 1980). The two pyrrodimethene units, however have considerable structural freedom about the $\mathrm{C}_{10^{-}}$ methylene group, which acts as a flexible hinge (Falk and Muller, 1983). A considerable body of spectroscopic evidence favours an intra-molecularly hydrogen bonded conformation for bilirubin in organic solvents, with two variants illustrated in figure 9. The structures proposed by Knell et al. (1972) and Kuenzle et al. (1973) vary only in the absence of hydrogen bonding due to the pyrrole $\mathrm{NH}$, in the case of the latter. The crystal structure of bilirubin (Bonnett et al., 1978) established a 'ridge-tile' geometry stabilized by an intramolecular hydrogen bonding pattern similar to that suggested by Knell et al. (1972). This conformation is also observed for di-isopropylammonium bilirubinate in the crystalline state (Mugnoli et al., 1978). A major feature of this structure (illustrated in figure 10) is that the two nearly planar dipyrromethene chromophores lie almost perpendicular to one another. The interplanar angles observed in the crystal structure for the two independent molecules are $\pm 96^{\circ}$ and $\pm 99^{\circ}$ (Bonnett et al., 1978). The $\mathrm{O}---\mathrm{O}$ distances between the propionate groups calculated from the published coordinates lie between 6.5 and $9 \cdot 9 \AA$. The

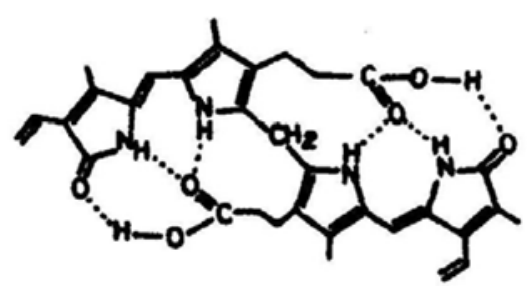

A

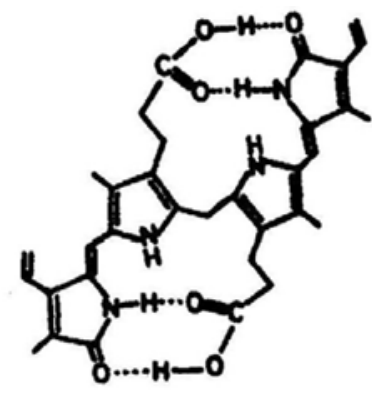

B

Figure 9. Proposed intramolecularly hydrogen-bonded structures for bilirubin-IX $\alpha$. Structure A is due to Knell et al.(1972) and B is from Kuenzle et al. (1973).
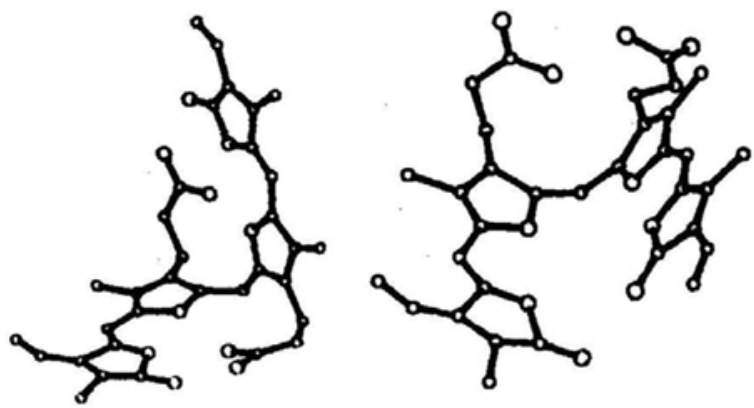

Figure 10. Perspective views of bilirubin conformations (left) 'ridge-tile' structure observed in crystals (Bonnett et al., 1978). Projection plotted using published coordinates (right). 'Quasi-cyclic' bilirubin conformation obtained for values of dihedral angles $\mathrm{N}_{22}-\mathrm{C}_{9}-$ $\mathrm{C}_{10}-\mathrm{C}_{11}(\Phi) \sim-80$ and $\mathrm{C}_{9},-\mathrm{C}_{10}-\mathrm{C}_{11}-\mathrm{N}_{23}(\Psi) \sim 60$. Note that the conformation represents 'left-handed' chirality. All other parameters are from the crystal structure of Bonnett et al. (1978) with the exception of the propionate sidechain dihedral angle $\mathrm{C}_{12}-\mathrm{C}_{35}-\mathrm{C}_{36}-\mathrm{C}_{37}$, which was fixed at $180^{\circ}$. Only $\mathrm{C}, \mathrm{N}, \mathrm{O}$ atoms are shown. 
$\mathrm{N}---\mathrm{N}$ distances calculated for the fully extended (all trans polymethylene chain) diamines, assuming idealized geometries, are $\mathrm{n}=23 \cdot 8 \AA, \mathrm{n}=46 \cdot 3 \AA, \mathrm{n}=57 \cdot 4 \AA$, $\mathrm{n}=68.8 \AA$ and $\mathrm{n}=811.3 \AA$. Permitting flexibility about the propionate sidechain, it appears that the $\mathrm{n}=6$ and $\mathrm{n}=8$ diamines can interact comfortably with a 'ridge-tile' bilirubin conformation. On the other hand the $\mathrm{n}=2$ diamine presumably forces bilirubin to adopt a conformation in which the propionate groups are significantly closer together.

We have used molecular modelling studies to generate a stereochemically allowed bilirubin conformation, which is 'quasi-cyclic' or 'porphyrin like' bringing the propionate sidechains into proximity. A steric map corresponding to rotations about the $\mathrm{C}_{9}-\mathrm{C}_{10}(\Phi)$ and $\mathrm{C}_{10}-\mathrm{C}_{11}(\Psi)$ bonds was generated using standard criteria for interatomic contacts (Ramachandran and Sasisekharan, 1968). For the crystal structure geometry the 'virtual dihedral angle' $\mathrm{C}_{2}-\mathrm{C}_{8}-\mathrm{C}_{12}-\mathrm{C}_{18}(\theta)$ was $\sim 97^{\circ}$, corresponding to a perpendicular arrangement of the dipyrromethene units. A search was made for allowed conformations with low values of $\theta$ i.e. between $0^{\circ}$ and $30^{\circ}$. For conformations with $\Phi \sim-80^{\circ}$ and $\Psi \sim 60^{\circ}$ a fully allowed structure with $\theta=-16^{\circ}$ can be obtained. A perspective view of this conformation is shown in figure 10. One of the propionate sidechain torsion angles has been altered to yield a fully extended sidechain. This bilirubin conformation is favourably disposed for simultaneous interaction of the propionate carboxylate groups with the two amino groups in the shorter diamines. The inter-propionate $\mathrm{O}---\mathrm{O}$ distances for the idealized conformations in figure 10 vary from 4.5 to $7 \cdot 6 \AA$. Flexibility about the propionate $\mathrm{C}-\mathrm{C}$ bonds can reduce these values further. The above analysis suggests that two distinct families of conformation are possible for bilirubin in dioxane solutions, depending on the nature of the interacting diamine. For $n \geq 6$ the classical 'ridge-tile' structure is favoured while for $\mathrm{n} \leq 4$ a 'quasi-cyclic' conformation appears suitable. It may be noted that both conformations are chiral and can result in high induced optical activity in complexes with chiral amines (Marr-Leisy et al., 1985).

The fluorescence results suggest that the 'ridge-tile' family of conformations has a high quantum yield while the 'quasi-cyclic' structures have low fluorescence. Dissipation of excitation energy is likely in the latter case by motions involving torsions about the $\mathrm{C}_{5}-\mathrm{C}_{6}$ and $\mathrm{C}_{14}-\mathrm{C}_{15}$ bonds. Quenching of fluorescence due to conformational mobility has indeed been suggested earlier (Lightner, 1982; Holzwarth, 1980). In chloroform and dichloromethane solutions the results suggest that the amines interact with a 'quasi-cyclic' bilirubin conformation, although the evidence is less than compelling. Nevertheless, the results of the present study provide further evidence that both solvent and the nature of the interacting receptor site can serve as conformational determinants for bilirubin.

\section{Acknowledgements}

We are grateful to Dr. Debra Marr-Leisy for carrying out some of the experiments described here and Dr. P. K. C. Paul for help with the molecular modelling studies.

\section{References}

Berthelot, P., Duvaldestin, Ph. and Fevery, J. (1982) in Bilirubin (eds K. P. M. Heirwegh and S. B. Brown) (Boca Raton, Florida: CRC Press) Vol. 2, p. 173. 
Bilirubin, G. (1983) Isr. J. Chem., 23, 201.

Blauer, G. and Wagniere, G. (1975) J. Am. Chem. Soc, 97, 1949.

Blauer, G., Lavie, E. and Silfen, J. (1977) Biochim. Biophys. Acta, 492, 64.

Bonnett, R., Davies, J. E., Hursthouse, M. B. and Sheldrick, G. M.(1978) Proc. R. Soc. London Ser. B., 202, 249.

Brodersen, R. (1980) CRC Crit. Rev. Clin. Lab. Sci, 11, 305.

Brodersen, R. (1982) in Bilirubin (eds K. P. M. Heirwegh and S. B. Brown) (Boca Raton, Florida: CRC Press) Vol. 1, p. 75.

Falk, H. and Muller, N. (1983) Tetrahedron, 39, 1875.

Furniss, B. S., Hannaford, A. J., Rugers, V., Smmyh, P. W. G. and Tatchell, A. R. (1978) Vogel's Textbook of Practical Organic Chemistry (London: Longman's) p. 274.

Geddes, A. J., Potterton, E. and Willis, G. (1980) Photochem. Photobiol, 34, 337.

Holzwarth, A. R., Langer, E., Lehner, H. and Schaffner, K. (1980) Photochem. Photobiol, 32, 17.

Knell, A. J., Johnson, B. and Hutchinson, D. W. (1972) Digestion, 6, 288.

Kuenzle, C. C, Weibel, M. H., Pelloni, R. R. and Hemmerich, P. (1973) Biochem. J., 133, 364.

Lightner, D. A. (1982) in Bilirubin (eds K. P. M. Heirwegh and S. B. Brown) (Boca Raton, Florida: CRC Press) Vol. 1, p. 1.

London, G. L., Park, Y. T. and Lightner, D. L. (1983) Tetrahedron, 39, 1893.

Marr-Leisy, D., Lahiri, K. and Balaram, P. (1985) Int. J. Peptide Protein Res., 25, 290.

McDonagh, A. F. and Assisi, F. (1972) Biochem. J., 129, 797.

Mugnoli, A., Manitto, P. and Monti, D. (1978) Nature (London), 273, 568.

Ramachandran, G. N. and Sasisekharan, V. (1968) Adv. Protein Chem., 23, 283. 